\title{
EDITORIAL
}

\section{Improving the delivery of coronary care for ethnic minorities}

\section{K Khunti, N J Samani}

Heart 2003;89:479-480

With the incidence of coronary heart disease being greater in certain ethnic communities in the UK, health professionals need to be aware of ethnic and cultural differences that may influence the presentation and behaviour of patients, in order to avoid potentially life threatening conditions being overlooked

7 he UK population is now diverse with nearly $7 \%$ of patients from the minority ethnic communities. ${ }^{1}$ The incidence and prevalence of coronary heart disease (CHD) is higher in many of these ethnic minority communities compared to their white counterparts. ${ }^{2}$ There have been repeated concerns raised about access to appropriate health care for patients from minority groups after CHD has been diagnosed. ${ }^{3}$ There are many possible reasons that could affect access and these need to be teased out if improvements are to occur.

In a recent issue of Heart, Barakat and colleagues $^{4}$ report an interesting observational study comparing the presentation and treatment of Bangladeshi and white patients admitted with acute myocardial infarction (MI) in east London. They found no significant differences between Bangladeshi and white patients in their own interpretation of the symptoms as being potentially MI and in the time from onset of chest pain to arrival in hospital. However, once the patients were in hospital it took almost twice as long on average (42.5 v 26 minutes) for the Bangladeshi patients to receive thrombolysis compared to the white patients. This was despite a greater proportion of the Bangladeshi patients being male, suffering from diabetes mellitus or having had a previous acute MI, which should have led to a greater suspicion of MI. Given the importance of rapid administration of thrombolytic treatment to appropriate patients and the emphasis put on this issue in the National Service Framework for coronary heart disease, ${ }^{5}$ the findings raise some concern.

See end of article for authors' affiliations

.........................

Correspondence to: Professor Nilesh J Samani, Division of Cardiology, University of Leicester, Clinical Sciences Wing Glenfield Hospital, Groby Road, Leicester, LE3 9QP, UK; njs@le.ac.uk
In keeping with previous studies, ${ }^{6}$ Barakat and colleagues show that South Asian people in the UK are as likely, if not more so, to seek immediate care for chest pain as white patients. ${ }^{4}$ Therefore, the delay in diagnosis is not caused by a different appreciation of patients from the ethnic minority community of the potential significance of their symptoms. However, an inability to communicate effectively with health care providers is a well

\section{COMMUNICATION BARRIER}

recognised and important barrier to health care delivery. ${ }^{78}$ This is again highlighted by the study of Barakat and colleagues. ${ }^{4}$ Only one third of the Bangladeshi patients could speak fluent English. Although, they do not discuss the extent to which interpreter facilities were available, it is likely that this had a significant impact on their findings.

\section{DIFFERENT MODES OF SYMPTOMS}

The more fascinating aspect of the study by Barakat and colleagues is their findings regarding the different modes of symptom presentation in the two ethnic groups. Thus, despite more of them having suffered a previous MI, Bangladeshi patients were less likely to describe classical features of ischaemic pain or localise the pain over the middle of the chest. To some extent, the variation in description of the pain may simply reflect different uses of specific terms-for example, "sharp" may be used to describe a more intense pain rather than the quality of the pain. There is also a culturally based tendency to "globalise" symptoms in some ethnic groups. For example, musculoskeletal pain is more widespread among South Asian ethnic minority communities in the UK compared to the white population. ${ }^{9}$ However, more curious and difficult to explain on either a cultural or physical basis is the different location of the pain in the two groups. In only $40 \%$ of the Bangladeshi patients was the chest pain centrally located compared with $87 \%$ in the white subjects; $34 \%$ of the Bangladeshi patients complained of left sided chest pain compared with only 3\% of the white subjects. While there are data showing that Asian and non-Asian patients vary in their knowledge of the anatomical location of the major body organs, ${ }^{10}$ this should not by itself affect the cutaneous location of a pain arising from an internal insult, and the basis of such inter-ethnic variations are not clear.

Notwithstanding this, it is of course very important that such differences in presentation are recognised. It is gratifying to note that in the study by Barakat and colleagues, ${ }^{4}$ once the diagnosis was made, there was no difference in the proportion of Bangladeshi and white patients given thrombolysis or treatment for secondary prevention. However, the study does not address the issue of whether the symptoms of more Bangladeshi patients were misinterpreted and more were discharged from casualty with a diagnosis of non-cardiac chest pain, and whether as a consequence their cared suffered. Further, while the study by Barakat and colleagues was carried out in the context of hospital presentation with acute chest pain, the findings have similar implications for other settings-general practice, rapid 
access chest pain clinics, hospital cardiology clinics-where patients with potential cardiac chest pain are assessed.

\section{PRACTICAL IMPLICATIONS}

So what are the practical implications of the findings of Barakat and colleagues? Those responsible for planning healthcare need to be aware of the ethnic composition of the patients within their area. To be able to provide effective care, health professionals need to be aware of ethnic and cultural differences that may influence presentation and behaviour, and discussion of these issues need to be incorporated in medical and nursing curricula. There is added benefit to patients from different cultures being able to consult in their own language ${ }^{11}$ and an ideal scenario would be bilingual staff. ${ }^{12}$ However, in recognition of the fact that this is not likely to be possible in many situations, provision of adequate interpretation facilities, not reliant on family members, would seem an essential requirement. By highlighting several of these issues, the study by Barakat and colleagues ${ }^{4}$ makes an important contribution. Not speaking English should not be a barrier to access to good quality care.

\section{Authors' affiliations}

K Khunti, Department of General Practice and Primary Health Care, University of Leicester, Leicester, UK

N J Samani, Division of Cardiology, University of Leicester

\section{REFERENCES}

1 Scott A, Pearce D, Gordblatt P. The sizes and characteristics of the minority ethnic populations of Great Britain - latest estimates. Population Trends 2001:105:6-15.

2 British Heart Foundation Coronary Heart Disease Statistics Database. London: British Heart Foundation, 2002. URL: www.bhf.org.uk

3 Hopkins A, Bahl V. Access to health care for people from black and ethnic minorities. London: Royal College of Physicians of London, 1993.

4 Barakat K, Wells Z, Ramdhany S, et al. Bangladeshi patients present with non-classic features of acute myocardial infarction and are treated ess aggressively in east London. Heart 2003:89:276-9.

5 Department of Healh. National service framework for coronary heart disease. London: Department of Health, 2000.

6 Chaturvedi N, Rai H, Ben Shlomo Y. Lay diagnosis and health-care-seeking behaviour for chest pain in south Asians and Europeans. Lancet 1997:350:1578-83.

7 Gerrish K. The nature and effect of communication difficulties arising from interactions between district nurses and South Asian patients and their carers. Journal of Advanced Nursing 2001;33:566-74.

8 Audit Commission. Testing times: a review of diabetes services in England and Wales. London: Audit Commission, 2000

9 Allison TR, Symmons DPM, Brammah T, et al. Musculoskeletal pain is more generalised among people from ethnic minorities than among white people in Greater Manchester. Ann Rheumatic Dis 2002:61:151-6.

10 Rashid A, Jagger C. Patients' knowledge of anatomical location of major organs within the human body: a comparison between Asians and non-Asians. Family Practice 1996;13:450-4.

11 Freeman GK, Rai H, Walker JJ, et al. Non-English speakers consulting with the GP in their own language: a cross-sectional survey. $\mathrm{Br} J \mathrm{Gen}$ Pract 2002;52:36-8.

12 Bhui K. Breaking down language barriers. The public favours bilingual staff over interpreters. BM 1998;317:817.

\section{IMAGES IN CARDIOLOGY}

\section{Pulmonary valve endocarditis}

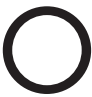

ne of the major applications of echocardiography is to identify vegetations in patients with suspected endocarditis. A 60 year old woman with sinus venosus atrial septal defect (ASD) presented with history of prolonged fever and dyspnoea. Before admission she was treated with several antibiotics, but without resolution of fever. Since the clinical suspicion of endocarditis was high a transthoracic echocardiogram was performed, which showed a large echodense mass on the pulmonic valve suggestive of vegetation (below left). Also noted was dilatation of the right sided cardiac chambers and pulmonary artery, and severe tricuspid and moderate pulmonary regurgitation suggestive of severe pulmonary hypertension. A transoesophageal echocardiogram confirmed these findings (below right) and revealed a large sinus venosus ASD with left to right shunt. Blood cultures remained negative, however, and the patient was treated empirically with

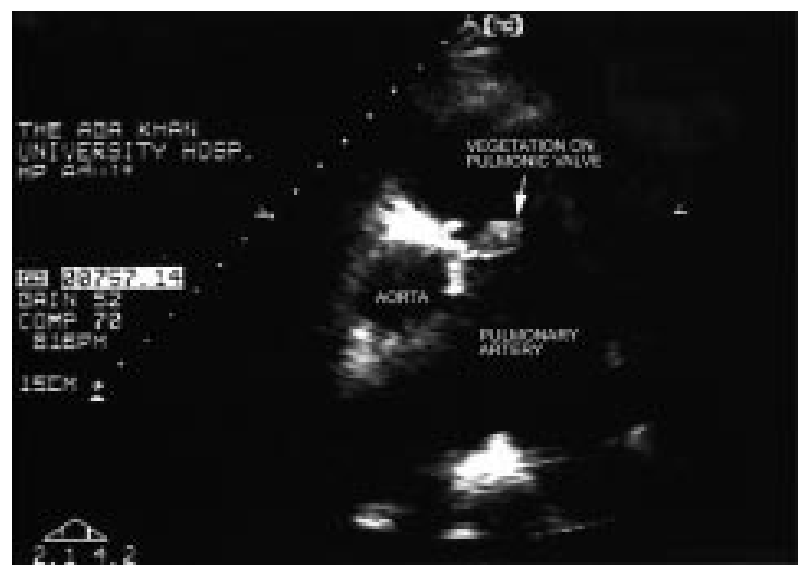

intravenous antibiotics. The hospital course was complicated by respiratory failure and pneumonia. Despite aggressive medical management the patient died in the hospital after a prolonged stay.

Isolated native pulmonary valve endocarditis is rare, accounting for $1.5-2.0 \%$ of all admissions for endocarditis. Intravenous drug abuse, alcoholism, sepsis, and catheter related infections account for the majority of predisposing factors. Between 1960 and 2000 only 38 cases have been reported in the literature in structurally normal valves. Intravenous antibiotics remain the primary mode of treatment, and indications for valve replacement are the same as for other valves.

F Jafary

saiid.dhakam@aku.edu

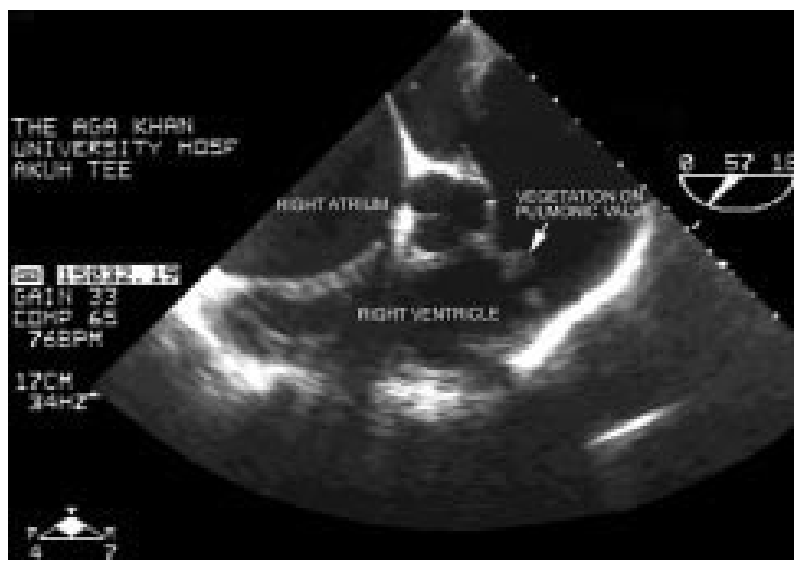

